\title{
Plexus Neuropathy in Infants Secondary to Arthro-Osteomyelitis: Report of 2 Cases and Review of the Literature
}

\author{
Vito Pavone ${ }^{\mathrm{a}}$, Maria Rosaria Politi ${ }^{\mathrm{b}}$, Michele Vecchio ${ }^{\mathrm{c}}$, Catia Romano ${ }^{\mathrm{b}}$, \\ Raffaele Falsaperla ${ }^{\mathrm{b}}$, Piero Pavone ${ }^{\mathrm{c}}$, Roberta Carboni ${ }^{\mathrm{d}}$, Vassilios Fanos ${ }^{\mathrm{d}, \mathrm{e}}$
}

\begin{abstract}
If the brachial nerves are affected in arthro-osteomyelitis, a flaccid palsy may result. Pediatricians and pediatric orthopedists should remain alert to signs of brachial palsy due a humeral infection, as this condition must be recognized rapidly and treated adequately. The cases of two infants who showed brachial palsy secondary to arthro-osteomyelitis, one of whom was followed up for seven years, were reported. Similar cases described in the literature were reviewed. Clinical features, absence of movement, and absence of biceps and triceps reflexes, were suggestive of peripheral brachial neuropathy: EEG and NCV investigations confirmed the diagnosis of true neuropathy. Early antibiotic treatment in these children resulted in rapid clinical improvement. According to our results and those reported in the literature, in infancy brachial palsy secondary to artro-osteomyelitis is uncommon; signs or symptoms of arthroosteomyelitis are often subtle and initially underestimated; arm flaccidity secondary to arthro-osteomyelitis may result in true paralysis (no pseudo-paralysis); invasive treatment may be prevented by a prompt diagnosis and antibiotic treatment.
\end{abstract}

Keywords: Arm flaccidity; Arthrocentesis; Arthro-osteomyelitis; True brachial palsy; Infantile brachial neuropathy

\section{Introduction}

While brachial palsy is a very common condition in the neo-

Manuscript accepted for publication October 4, 2012

${ }^{a}$ Department of Orthopedics, University-Hospital OVE-Policlinic, University of Catania, Italy

${ }^{\mathrm{b}}$ Department of Pediatric and Pediatric Emergency, University-Hospital OVE-Policlinic, University of Catania, Catania, Italy

${ }^{c}$ Department of Neurophysiology, University-Hospital OVE-Policlinic, University of Catania, Italy

${ }^{\mathrm{d}} \mathrm{NICU}$, Puericulture Institute and Neonatal Section, University of Cagliari, Italy

${ }^{\mathrm{e}}$ Corresponding author: Vassilios Fanos, Chief Neonatal Intensive Care Unit, Puericultura Institute and Neonatal Section, Department of

Surgery Sciences-University of Cagliari, Via Ospedale, 119 - 09124

Cagliari, Italy. Email: vafanos@tiscali.it

doi: http://dx.doi.org/10.4021/ijcp60w natal period, with an incidence ranging from 0.6 to 4.6 per 1,000 live births, it can also manifest, albeit more rarely, during the subsequent months [1-9]. Infant brachial palsy is related to stretching, conditions that exert pressure or infectious events. These events can injure the brachial plexus, resulting in the involvement of the upper part of the arm or, more rarely, the forearm and hand.

Arthro-osteomyelitis occurs rarely in the neonatal [1012] and infantile periods and is mostly due to bacterial infections, mainly with Staph. aureus, Group-B Streptococcus, and E. coli. Brachial plexus palsy has been reported to occur after osteomyelitis and arthritis [1-9] and thus neonatologists must remain alert to the possibility that brachial palsy may disguise an infection potentially associated to severe bone impairment, namely in case of late diagnosis.

The cases of two patients admitted to the Vittorio Emanuele Hospital Authority-University-Policlinico, Catania, Italy who was affected by brachial plexus neuropathy documented by EMG and NCV, secondary to arthro-osteomyelitis are reported here. One of them was followed up for more than seven years. The cases of brachial palsy secondary to arthroosteomyelitis described in the literature are also reviewed.

\section{Case Report}

\section{Case 1}

A male infant aged three months and 21 days was admitted to our Department after the parents observed that he did not move his right arm. The family history was uneventful. The infant was the second-born child of healthy non-consanguineous parents. The mother stated that she had not had any infectious diseases or taken any drugs during the pregnancy. The baby was born at term by cesarean section, due to a cervical dystocia, with a weight of $3,340 \mathrm{~g}$, a length of $51 \mathrm{~cm}$, and a head circumference of $35 \mathrm{~cm}$. The neonatal period was uneventful. The baby was discharged from the hospital three days after birth. The infant did not manifest any physical problems and the movements of all limbs appeared normal until two and a half months after birth, when the infant had an episode of bronchiolitis that lasted about a week. A month 


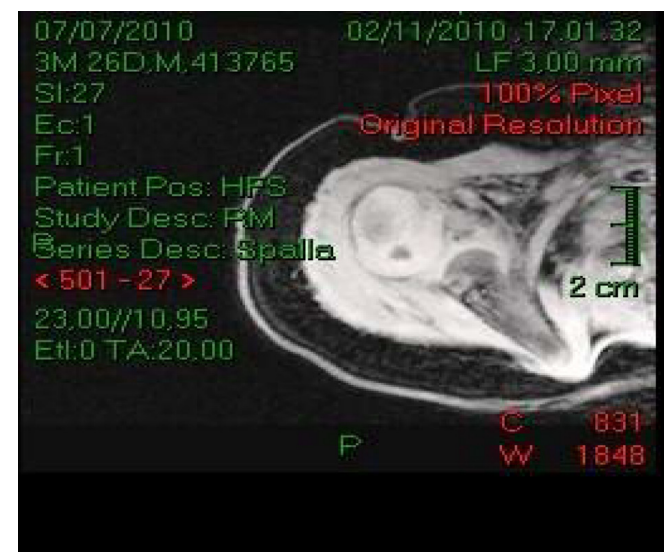

Figure 1. Magnetic resonance imaging of the right shoulder in Case 1 revealed effusion and necrotic areas at the humeral head that were suggestive of septic arthritis.

later, the infant had a febrile episode that lasted 5 days. This was followed by the loss of movement in the right arm, associated irritability, and crying when the right arm and shoulder were touched or moved.

On admission, the infant was afebrile and his weight, length and head circumference were within the normal limits. Active movements of the right arm were absent and the arm was flaccid and adducted with internal rotation of the arm and pronation of the forearm and hand. There was swelling of the right shoulder and passive movements caused pain, crying and irritability. Right biceps and triceps reflexes were absent but sensation appeared to be preserved. The Moro's reflex was asymmetric. No other abnormalities were observed during the physical examination. Laboratory analyses revealed a white blood cell count (WBC) of $11.340 / \mathrm{mm}^{3}$ with $40 \%$ neutrophils, an increased erythrocyte sedimentation rate (ESR) of $111 \mathrm{~mm} / \mathrm{h}$, and high C-reactive protein levels $(72.9 \mathrm{mg} / \mathrm{dL})$. The blood culture was negative, as were the other laboratory tests. Arthrocentesis culture disclosed Group-B Streptococcus.

Orthopedic consultation confirmed the absence of motility of the right arm, discomfort after passive movements, and the presence of a mild palpable mass on the shoulder. Ultrasonography of the right shoulder revealed the presence of a fluid pouch that was $6 \mathrm{~mm}$ in size in the medial articular recess and a hypo-anechoic layer $(3 \mathrm{~mm})$ peripheral to the biceps tendon. Magnetic resonance imaging (MRI) of the right shoulder and limb showed significant joint effusions in the shoulder and the presence of three circular osteolytic areas on the head of the humerus, glenoid and coracoid apophysis that were suggestive of an osteo-articular septic process (Fig. 1).

The child also underwent nerve conduction studies and electromyography (EMG) on the 12th day after admission. EMG of the right deltoid and biceps showed a reduction of recruitment patterns and demyelination without axonal loss of the biceps and deltoid myotomes along with conduction block of the accessory nerve upon stimulation of Erb's point. This report was compatible with a brachial plexus injury.

The clinical features, laboratory findings, and radiological signs together indicated a diagnosis of arthro-osteomyelitis with secondary right brachial palsy. The patient was administered antibiotic therapy with intravenous cefazolin $(100 \mathrm{mg} / \mathrm{kg}$ per day) for 20 days and the right arm was immobilized with a Desault bandage. After the antibiotic treatment was completed, the light swelling disappeared. Oral amoxicillin-clavulanate treatment was then administered for another 3 weeks and physiotherapy was started.

A shoulder X-ray, taken 10 days after the cefazolin treatment was completed, revealed an irregularity of the bone profile in the iuxta-metaphyseal region of the proximal diaphysis of the right humerus. However, the clinical conditions and right limb palsy improved rapidly and the inflammatory signs normalized one month after admission. Aspiration and surgical drainage was not necessary. The child was discharged when he had recovered almost completely.

Thirty days after discharge, the child came back for follow up. A physical examination revealed the presence of normal passive and active movements of the right shoulder. The Moro's reflex had become symmetric and deep tendon reflexes were present. Laboratory studies revealed that all abnormalities, particularly the inflammatory indices and WBC, had returned to normal. Echography of the shoulder revealed it was normal and EMG showed a normal recruitment pattern of the deltoid and biceps. MRI of the right shoulder performed at 1 years of age was normal.

\section{Case 2}

A 3-week-old female was referred to our Department due to hypomotility of the right arm. The infant had been born at term with a normal weight, length and head circumference. The family history was unremarkable and the pregnancy and delivery were reported to have been uncomplicated. The mother reported that the infant had fallen off the bed a week earlier and had a trauma of the upper right limb. No signs of child abuse were noticed. However, an X-ray of the shoulder on admission failed to detect any abnormalities and the infant was discharged from the hospital. Ten days later, the infant was admitted for the second time because of loss of active movements of the upper right arm and the presence of irritability, fever, and pain when the right arm was moved passively. The baby weighed $6,000 \mathrm{~g}$, was $63 \mathrm{~cm}$ long, and had a head circumference of $42 \mathrm{~cm}$. All of these measurements were within the normal limits. The infant was febrile with a temperature of $38.2{ }^{\circ} \mathrm{C}$ and showed signs of right shoulder involvement, namely warm swelling skin and pain upon passive movements of the arm. The right arm was flaccid, adducted and internally rotated with the forearm pronated. There was no hand grasp. The deep tendon reflexes 
of the biceps and triceps were absent but sensation was not affected.

Laboratory findings revealed an ESR of $98 \mathrm{~mm} / \mathrm{h}$, a WBC of $14.000 / \mathrm{mm}^{3}$ with $80 \%$ polymorphonuclear cells, and high C-reactive protein levels $(74 \mathrm{mg} / \mathrm{dL})$. Serum immunoglobulin levels were normal. The blood culture revealed an infection of Group-B Streptococcus. The X-ray showed notable disruption of the proximal metaphysis. An EMG revealed abnormalities with reduced recruitment of motor units and showed decreased sensory amplitudes of the median and ulnar nerves in the right upper extremity as compared with those in the left. MRI confirmed the anomaly observed in the second X-ray; it also revealed diffuse marrow and periosteal signal changes that were consistent with arthro-osteomyelitis. The patient underwent antibiotic treatment with intravenous cefotaxime for 21 days and her right arm was immobilized with a Desault bandage for three weeks. Surgical treatment was not deemed necessary. Amoxicillin-clavulanate was administered orally for 3 weeks after the cefotaxime treatment had been completed. The patient underwent physiotherapy. Recovery was reported at 3 months later.

At the age of 5 months, the child underwent a Tc-99m body scan that revealed resolution of the inflammation process. Follow up at 2, 4 and 7 years of age revealed the arms moved normally. X-ray at 5 years of age revealed a normal length of arms, with a residual slight irregular morphology of the right humeral epiphysis compared to the contralateral shoulder.

\section{Discussion}

The two patients described here developed brachial plexus palsy in the first four months of life secondary to an infectious event that involved the shoulder joint and humeral bone. Brachial palsy occurs frequently in newborns, particularly when the birth weight is high and delivery is complicated by presentation anomalies. The paralysis is flaccid and localized in the arm and affects different nerves of the brachial plexus (C5 to T1). The upper root is most commonly involved, which results in paralysis or weakness of the shoulder, elbow and wrist (C5-C6: Erb-Duchenne paralysis). The extension of the injury to the 7 th and 8 th cervical nerves and 1 st thoracic nerve causes paralysis of the entire arm and related disorders (Klumpke paralysis-Horner syndrome). Brachial palsy can also result from compressive events at the level of the plexus and/or infectious events.

Osteomyelitis is unusual in infants. It is caused by bacteria that either have a hematogenous origin or are inoculated directly into the bone; previous minor closed trauma is a predisposing factor. In Case 2, the predisposing factor was a traumatic event. In early onset osteomyelitis, concurrent arthritis is quite frequent. This is because transphyseal blood vessel continuity between the metaphysis and epiphysis al- lows the infection to reach the joint space and vice versa. Studies have shown that 76\% [12], 70\% [13], 47\% [14], and $28 \%$ [15] of osteomyelitis cases are associated with septic arthritis.

In infants, the signs or symptoms of arthro-osteomyelitis are usually not specific. In addition, the WBC and ESR may be normal or slightly increased. Fever, irritability, pain, refusal to feed, and a poor general condition may also not be observed. In fact, in infants, the earliest signs and symptoms of infections are often subtle and the laboratory findings may not be diagnostic. Indeed, in both our patients, the most impressive signs that led us to a diagnosis were a slight swelling of the right shoulder and pain during passive movements.

It remains unclear whether brachial plexus palsy secondary to arthro-osteomyelitis should be regarded as a pseudoparalysis secondary to pain or a true neuropathy. We believe that both of these conditions may be true. The differential diagnosis between true paralysis and pseudo-paralysis is based on clinical and neurophysiological findings. In pseudo-paralysis, the movement causes pain but movement is still possible, even if it is only partial; the biceps and triceps reflexes are present; EMG investigations are normal; and the recovery is rapid. In true paralysis, the arm is flaccid; biceps and triceps reflexes are absent and EMG reveals signs of denervation. In our patients, the clinical signs were associated with typical aspect of neuropathy (flaccid arm, positivity of EMG with a recruitment pattern, and absence of reflexes).

The pathophysiological process involved in brachial plexus neuropathy secondary to arthro-osteomyelitis is not quite clear. Clay [1] advanced the hypothesis that brachial plexus neuropathy may be due to nerve ischemia secondary to thrombophlebitis and occlusion of the vasa nervorum.

The published literature suggests that brachial palsy secondary to arthro-osteomyelitis is quite rare. Table 1 (supplemental data, www.ijcp.elmerpress.com) summarizes the details of the 17 cases apart from the two described here of brachial palsy secondary to arthro-osteomyelitis that have been reported in the literature. The age of the patients ranged from 3 - 90 days, the type of delivery was dystocic in 4 cases, and in 8 cases the delivery was vaginal. The right sides were more frequently affected. The main symptoms that were reported were fever, pain and swelling of the shoulder. In most cases, the laboratory findings revealed increased WBC and ESR. The EMG showed clear signs of denervation in all of the cases where it was performed. The electrophysiological pattern usually showed a severe attenuation of the amplitude of motor and sensitive nerve action potentials evoked or recorded below the damaged area, and a variable slowing of motor and sensory conduction across the damaged area. Xrays were useful for showing the impairment of the shoulder and metaphysis and epiphysis but MRI should be considered to be the best tool for this purpose since Bonhoeffer et al [16] have found that it is sensitive enough to detect early osteomyelitis in $92 \%$ of cases. 
In the literature the treatment depended on the degree of damage and consisted of antibiotics to treat the infection and prevent the destruction of the articular cartilage and the spread of the microorganisms to other areas. The antimicrobial therapy is advised to be administered intravenously and continued for at least 3 weeks until the clinical and laboratory investigations revealed improvements. This treatment is then followed by oral therapy until the patient had recovered. The orthopedic treatments consisted of surgical drainage from the joint and bone when indicated. Arthrocentesis was performed in 11 out of the 17 cases but was not deemed necessary in the remaining cases. However, arthrocentesis was useful for identifying the bacteria that were involved in arthro-osteomyelitis pathogenesis. These included Group-B Streptococcus (seven cases), Staph. aureus (three cases), and Klebsiella spp., Enterobacter spp., Str. viridans and Gram positive, one case.

The prognosis was good in most cases with the exception of Case 2 of Clay [1], where the recovery took 12 months; Case 2 of Isaacs et al [2], where recovery took an unspecified long period; and the case described by Gabriel et al [4], where weakness and partial recovery were still present at the age of 3 years. However, a complete recovery was obtained in all remaining cases within six months. Case 1 in the present study exhibited a complete recovery two months after onset and Case 2 after three months. In Case 2, where follow up continued for 7 years, there was a persistence of a mild radiological anomaly that consisted of slight irregular morphology of the humeral epiphysis compared to the contralateral side.

Arm flaccidity secondary to arthro-osteomyelitis reported in the two patients resulted in transient true neuropathy, and not pseudo-paralysis. Prompt diagnosis and rapid improvement with antibiotic treatment avoided invasive treatment.

We wish to emphasize that brachial palsy secondary to arthro-osteomyelitis may go unnoticed in infants. It is important that neonatologists, pediatricians and pediatric orthopedists remain alert to signs of this condition because, while the outcome is favorable in most cases, a delay in antibiotic treatment can result in damage to the arm with severe sequelae. It is important to choose the right antibiotic for the right patient [16-18].

\section{Conclusions}

In infancy, asymmetric arm flaccidity may be secondary to an infective event affecting the bone. However, according to our experience and the results reported in the literature, brachial palsy secondary to artro-osteomyelitis is uncommon; signs or symptoms of arthro-osteomyelitis are often subtle and could be underestimated; arm flaccidity secondary to arthro-osteomyelitis may result in true paralysis; a prompt diagnosis and antibiotic treatment may prevent invasive sur- gical treatment and severe outcome.

\section{References}

1. Clay SA. Osteomyelitis as a cause of brachial plexus neuropathy. Am J Dis Child. 1982;136(12):1054-1056.

2. Isaacs D, Bower BD, Moxon ER. Neonatal osteomyelitis presenting as nerve palsy. Br Med J (Clin Res Ed). 1986;292(6527):1071.

3. Wang YC, Lin FK, Hung KL, Wu DY. Brachial plexus neuropathy secondary to septic arthritis and osteomyelitis: report of two cases. Zhonghua Min Guo Xiao Er Ke Yi Xue Hui Za Zhi. 1994;35(5):449-454.

4. Gabriel SR, Thometz JG, Jaradeh S. Septic arthritis associated with brachial plexus neuropathy. A case report. J Bone Joint Surg Am. 1996;78(1):103-105.

5. Sadleir LG, Connolly MB. Acquired brachial-plexus neuropathy in the neonate: a rare presentation of lateonset group-B streptococcal osteomyelitis. Dev Med Child Neurol. 1998;40(7):496-499.

6. Smida M, Jalel C, Soohun T, Djebbi Y, Nessib MN, Ben Ghachem M. [Brachial plexus paralysis of infectious origin: report of four cases]. Arch Pediatr. 2002;9(8):814817.

7. Solebo JO, Keane MR, Obaro RO, Browne LM. Osteomyelitis of head of humerus presenting as Erbs palsy in a neonate. Eur J Pediatr. 2004;163(4-5):262.

8. Liao SL, Lai SH, Lin TY, Chou YH, Hsu JF. Premature rupture of the membranes: a cause for neonatal osteomyelitis? Am J Perinatol. 2005;22(2):63-66.

9. Estienne M, Scaioli V, Zibordi F, Angelini L. Enigmatic osteomyelitis and bilateral upper limb palsy in a neonate. Pediatr Neurol. 2005;32(1):56-59.

10. Chung T. Magnetic resonance imaging in acute osteomyelitis in children. Pediatr Infect Dis J. 2002;21(9):869870.

11. Ceroni D, Regusci M, Pazos J, Dayer R, Kaelin A. [Acute bone and joint infections in children: how much attention should be paid to persistent fever during intravenous antibiotic therapy?]. Rev Chir Orthop Reparatrice Appar Mot. 2003;89(3):250-256.

12. Caramia G, Ruffini E, Marco Zaffanello M, Fanos V. Acute bone and joint infections in children and therapeutic options. Pediatr Infec Dis J. 2007; 2:4:193-203.

13. Halder D, Seng QB, Malik AS, Choo KE. Neonatal septic arthritis. Southeast Asian J Trop Med Public Health. 1996;27(3):600-605.

14. Wong M, Isaacs D, Howman-Giles R, Uren R. Clinical and diagnostic features of osteomyelitis occurring in the first three months of life. Pediatr Infect Dis J. 1995;14(12):1047-1053.

15. Asmar BI. Osteomyelitis in the neonate. Infect Dis Clin North Am. 1992;6(1):117-132. 
16. Bonhoeffer J, Haeberle B, Schaad UB, Heininger U. Diagnosis of acute haematogenous osteomyelitis and septic arthritis: 20 years experience at the University Children's Hospital Basel. Swiss Med Wkly. 2001;131(39-40):575581.
17. Fanos V, Dall'Agnola A. Antibiotics in neonatal infections: a review. Drugs. 1999;58(3):405-427.

18. Fanos V, Cuzzolin L, Atzei A, Testa M. Antibiotics and antifungals in neonatal intensive care units: a review. $\mathrm{J}$ Chemother. 2007;19(1):5-20. 